\title{
Diacronie
}

Studi di Storia Contemporanea

$\mathrm{N}^{\circ} 28,4 \mid 2016$

La voce del silenzio

\section{Gabriel Gorodetsky (edited by), The Maisky Diaries: Red Ambassador to the Court of St James's 1932-1943}

\section{Claudia Baldoli e Luigi Petrella}

\section{(2) OpenEdition}

Edizione digitale

URL: http://journals.openedition.org/diacronie/4537

DOI: 10.4000/diacronie.4537

ISSN: 2038-0925

Editore

Association culturelle Diacronie

Notizia bibliografica digitale

Claudia Baldoli e Luigi Petrella, « Gabriel Gorodetsky (edited by), The Maisky Diaries: Red Ambassador to the Court of St James's 1932-1943 », Diacronie [Online], N²8, 4 | 2016, Messo online il 29 décembre 2016, consultato il 24 septembre 2020. URL : http://journals.openedition.org/diacronie/4537 ; DOI : https://doi.org/10.4000/diacronie.4537 


\title{
Diacronie
}

N. 28 | 4|2016 La voce del silenzio: intelligence, spionaggio e conflitto nel XX secolo

21/

\section{PANORAMICA: Gran Bretagna 2015}

\author{
Claudia BALDOLI, Luigi PETRELLA*
}

GORODETSKY, Gabriel (edited by), The Maisky Diaries: Red Ambassador to the Court of St James's 1932-1943, New Haven-London, Yale University Press, 2015, 632 pp.

a cura di Claudia BALDOLI, Luigi PETRELLA

In attesa che arrivino in libreria i tre volumi della versione integrale annotata, la pubblicazione della selezione dei diari di Ivan Maisky, ambasciatore dell'Unione Sovietica a Londra dal 1932 al 1943, ha reso disponibile per la prima volta in inglese una fonte straordinaria per lo studio della politica britannica e delle relazioni internazionali in un decennio cruciale della storia europea e mondiale, scandito da passaggi cruciali come l'illusione dell'appeasement, i negoziati che condussero alla firma del patto Molotov-Ribbentropp, l'ascesa di Churchill al governo, l'invasione nazista della Russia e l'acceso dibattito sull'apertura del secondo fronte. Il curatore Gabriel Gorodetsky, fellow dell'All Souls College di Oxford e professore emerito di storia all'università di Tel Aviv, scoprì i documenti originali negli archivi moscoviti nel 1993.

Discendente da una famiglia ebreo-polacca ed esule dalla Russia zarista, estroverso e mondano, Maisky aveva visitato Londra per la prima volta nel 1912 e otto anni dopo era entrato nella diplomazia del nuovo stato rivoluzionario, uscendo miracolosamente indenne dalle purghe degli anni Trenta, a cui lo candidavano la sua reputazione di intellettuale cosmopolita e un'eccezionale indipendenza di spirito e di giudizio. Il lungo soggiorno a Londra e il canale diretto che era stato in grado di aprire con i maggiori politici inglesi lo resero indispensabile al suo governo negli anni in cui la comprensione della politica estera britannica, in primo luogo nei confronti della Germania nazista, era 
vitale per Mosca. Il diario getta luce anche sul ruolo che le rivalità interne al Cremlino giocarono nella formulazione della politica estera sovietica negli anni Trenta.

Per più di dieci anni, il diplomatico riuscì a intrattenere una fittissima rete di rapporti con le figure più in vista della vita pubblica britannica, senza peraltro raggiungere lo scopo di contrastare l'indisponibilità, prevalente nell'establishment di Londra, a prendere di petto Hitler e Mussolini fin dal 1938. Le pagine dei suoi diari registrano le lunghe conversazioni che Maysky ebbe regolarmente con politici come Winston Churchill, Anthony Eden, Lord Halifax e David Lloyd George, editori come Lord Beaverbrook, diplomatici come Joseph Kennedy (ambasciatore degli Stati Uniti a Londra dal 1938 al 1940), e intellettuali come Sidney e Beatrice Webb, John Maynard Keynes, H. G. Wells e George Bernard Shaw.

CADBURY, Deborah, Princes at War: The British Royal Family's Private Battle in the Second World War, London, Bloomsbury Publishing, 2015, 432 pp.

a cura di Claudia BALDOLI, Luigi PETRELLA

Le vicende della famiglia reale inglese durante la Seconda guerra mondiale sono al centro del libro di Deborah Cadbury, scrittrice di storia pluripremiata e per trent'anni producer della BBC. Il suo lavoro parte dalla crisi che condusse nel 1936 all'abdicazione di Edoardo VIII, che rinunciò al trono per sposare Wallis Simpson, e si snoda negli anni della crescente minaccia nazista in Europa. Il fratello minore Albert, che divenne re con il nome di Giorgio VI, pur afflitto dalla balbuzie e da un carattere timido e insicuro, inizialmente riluttante ad accettare la responsabilità del trono, ma alla fine rassegnato al suo destino, si trovò a fare i conti con le divisioni interne alla famiglia reale e con gli imbarazzanti comportamenti del Duca di Windsor e della moglie, i cui orientamenti disfattisti suscitavano sospetti perfino di intese con il nemico. Attingendo a nuove fonti, l'autrice indaga sui retroscena di Buckingham Palace e spiega come fu possibile alla monarchia contrastare un declino che appariva inevitabile, assecondando la scelta di entrare in guerra contro Hitler e mostrando di condividere i sacrifici e le privazioni della gente comune. 


\section{CORTON, Christine L., London Fog: The Biography, Harvard (MA)-} London, Harvard University Press, 2015, 408 pp.

a cura di Claudia BALDOLI, Luigi PETRELLA

Christine Corton, fellow del Wolfson College di Cambridge, ha dedicato un originale lavoro biografico di grande successo, anche popolare, alla nebbia, elemento distintivo degli inverni londinesi per settecento anni, dalle sue origini nel tredicesimo secolo al suo progressivo esaurimento nella seconda metà del ventesimo. La combustione del carbone su larga scala inizialmente solo per usi domestici e poi, dalla rivoluzione industriale, per la meccanizzazione della produzione - combinata con le basse temperature, l'assenza di vento, la morfologia e l'idrografia dell'area londinese produsse un fenomeno che oltre a causare enormi costi sociali influenzò profondamente abitudini collettive, relazioni sociali, creazioni letterarie e artistiche. Solo i cinque giorni di "smog killer" del 1952, che causarono direttamente o indirettamente la morte di 4.000 persone, aprirono la strada a una legislazione che quattro anni dopo avviò la conversione dei londinesi all'uso di gas ed elettricità per il riscaldamento e incentivò l'impiego a scopi industriali di combustibili più economici e meno inquinanti del carbone.

PEDERSEN, Susan, The Guardians: The League of Nations and the Crisis of Empire, Oxford, Oxford University Press, 2015, 592 pp.

a cura di Claudia BALDOLI, Luigi PETRELLA

Susan Pedersen (docente di storia alla Columbia University) ha dedicato un libro al sistema dei mandati fiduciari in cui sostiene che il giudizio storico sulla Società delle Nazioni, travolta dall'ascesa dei fascismi in Europa e incapace di impedire la Seconda guerra mondiale, è stato troppo sbrigativo e severo. Il lavoro - che estende il suo raggio di analisi alle vicende di quattro continenti e si basa su una mole impressionante di fonti d'archivio - non sottovaluta i limiti più gravi dell'esperimento internazionalista ma, ricostruendo in particolare le vicende della Commissione permanente e il ruolo, su tutti, del rappresentante britannico James Drummond (primo segretario della Società delle Nazioni, dal 1919 al 1933), mette in luce i passi avanti compiuti nel tentativo di smarcare l'organizzazione dalla pressione degli interessi nazionali. Pur non potendo impedire alle potenze mandatarie di controllare le ex colonie tedesche e le aree mediorientali già appartenute all'Impero ottomano con i metodi che preferivano (nel 1925 la Francia, impegnata a neutralizzare i nazionalisti siriani, bombardò il centro di 
Damasco), la Commissione esercitò pressioni per attenuare i tratti più duri della loro amministrazione, per esempio nel trattamento delle minoranze, suscitando un dibattito pubblico sull'ipotesi che i governi nazionali potessero rispondere a un organismo internazionale.

SEATON, Jean, Pinkoes and Traitors: The BBC and the Nation, 1974-1987, London, Profile Books, 2015, 384 pp.

a cura di Claudia BALDOLI, Luigi PETRELLA

Il lavoro di Jean Seaton, che insegna storia dei mass media all'università di Westminster, è dedicato alle vicende della $\mathrm{BBC}$ in uno dei periodi più delicati della recente storia britannica, che coincide in larga parte con il governo di Margaret Thatcher e più ampiamente con l'influenza delle idee economiche e sociali portate in auge dall'egemonia dei conservatori in poco meno di un quindicennio. La tesi di fondo dell'autrice è che la radiotelevisione pubblica britannica abbia risposto con una "rivoluzione creativa" ai tentativi di ridimensionamento avviati dal governo laburista alla metà degli anni Settanta, soprattutto con l'ipotesi di abolire l'abbonamento annuale pagato dal pubblico, e condotti con maggior vigore nel decennio successivo prospettando l'ipotesi della privatizzazione. In particolare, Seaton ricostruisce gli sforzi compiuti dagli apparati della BBC, anche con negoziati e compromessi con gli interlocutori politici meno ostili, per conservare alla missione di servizio pubblico una sfera di imparzialità e di originalità nell'attività di informazione e nella realizzazione dei programmi di successo presso il grande pubblico.

\section{URBACH, Karina, Go-Betweens for Hitler, Oxford, Oxford University Press, 2015, 408 pp. \\ a cura di Claudia BALDOLI, Luigi PETRELLA}

Karina Urbach, senior research fellow all'Institute of Historical Research di Londra, ricostruisce la storia degli aristocratici tedeschi che agirono da intermediari ufficiosi tra Hitler e le altre potenze europee negli anni Trenta e durante la guerra. Una storia della diplomazia segreta, che offre nuovi elementi soprattutto per l'analisi della politica di appeasement, ricostruita su fonti inesplorate, scovate non solo negli archivi tedeschi e britannici, ma anche in Olanda, nella Repubblica Ceca e negli Stati Uniti. L'autrice mette in luce quanto questa rete informale dovesse alla natura peculiare della diplomazia prima della Grande Guerra, dominata dall'aristocrazia e dagli intrecci 
familiari che spesso valicavano i confini nazionali. La figura centrale del libro è Carl Eduard, duca di SachsenCoburg und Gotha, inglese di nascita, pronipote della regina Vittoria, che per vicende di successione fu posto all'età di 15 anni alla guida di uno staterello tedesco. Nella prima guerra mondiale combatté contro gli inglesi, che per questo lo privarono del titolo. Vent'anni dopo, Hitler ne fece uno dei protagonisti del suo tentativo di conquistare la benevolenza delle élite di Londra. 


\section{* Gli autori}

Claudia Baldoli è Senior Lecturer in European History presso la University of Newcastle. È autrice di Exporting fascism: Italian fascists and Britain's Italians in the 1930s, Oxford-New York, Berg, 2003 e curatrice (assieme ad Andrew Knapp e Richard Overy) di Bombing, states and peoples in Western Europe, 1940-1945, London-New York, Continuum, 2011 e (con Adrew Knapp) Forgotten blitzes : France and Italy under Allied air attack, 1940-1945, London-New York, Continuum, 2012.

URL: < http://www.studistorici.com/progett/comitato-scientifico/ >

Luigi Petrella ha conseguito il dottorato di ricerca in Storia presso la University of Newcastle, dove è teaching assistant. In precedenza ha lavorato come giornalista per venticinque anni. 亡̀ autore di Staging the Fascist War: The Ministry of Popular Culture and Italian Propaganda on the Home Front, 1938-1943, Oxford-Bern-Berlin-Bruxelles-Frankfurt am Main-New York-Wien, Peter Lang, 2016..

URL: < http://www.studistorici.com/progett/autori/\#Petrella >

\section{Per citare questo articolo:}

BALDOLI, Claudia, PETRELLA, Luigi, «Panoramica: Gran Bretagna 2015», Diacronie. Studi di Storia Contemporanea : La voce del silenzio: intelligence, spionaggio e conflitto nel XX secolo, 29/12/2016,

URL:< http://www.studistorici.com/2016/12/29/gran-bretagna_numero_28/ >

Diacronie Studi di Storia Contemporanea 3 www.diacronie.it

Risorsa digitale indipendente a carattere storiografico. Uscita trimestrale. redazione.diacronie@hotmail.it

Comitato di redazione: Jacopo Bassi - Luca Bufarale - Antonio César Moreno Cantano - Deborah Paci - Fausto Pietrancosta - Alessandro Salvador - Matteo Tomasoni - Luca Zuccolo

Diritti: gli articoli di Diacronie. Studi di Storia Contemporanea sono pubblicati sotto licenza Creative Commons 3.0. Possono essere riprodotti e modificati a patto di indicare eventuali modifiche dei contenuti, di riconoscere la paternità dell'opera e di condividerla allo stesso modo. La citazione di estratti è comunque sempre autorizzata, nei limiti previsti dalla legge. 